\title{
Image Segmentation Techniques and its applications for Knee Joints: a Survey
}

\author{
Meenaz H. Shaikh ${ }^{1}$, Shraddha Panbude ${ }^{2}$, Anuradha Joshi ${ }^{3}$ \\ ${ }^{I}$ Electronics \& Telecommunication, Mumbai University, India \\ ${ }^{2}$ Electronics \& Telecommunication, Mumbai University, India \\ ${ }^{3}$ Electronics \& Telecommunication, Mumbai University, India
}

\begin{abstract}
Knee joint is the largest anatomical joint within the human body which facilitates the ease of movement from one place to another. Osteoarthritis (OA), a common debilitating disease afflicting over 71 million people globally, is poorly understood and a treatment to slow, halt, or reverse the disease progression remains elusive. It is characterized by the degeneration of the articular cartilage in joints. Image segmentation is the fundamental step to analyze images and extract data from them. Segmentation process also helps to find region of interest in a particular image. This paper addresses various image segmentation techniques, evaluating them and presents the characteristics related to those techniques. Also the segmentation of knee joint and future work are mentioned.
\end{abstract}

Keywords - Image Segmentation, knee, knee segmentation, Osteoarthritis.

\section{INTRODUCTION}

Osteoarthritis (OA) is one of the major health concerns among the elderly today. The main effects of $\mathrm{OA}$ is the degradation of the articular cartilage together with remodeling and overgrowth of bone, a process causing loss of mobility of the joints. MRI allows for quantitative evaluation of the cartilage and cartilage deterioration can be detected using this technique. MRI also has the advantage of being a non-invasive technique. When assessing the cartilage, the MR scans can be manually segmented slice-by-slice by experts, but for clinical studies manual methods are too time consuming and are also prone to inter- and intra-observer variability [1]. When automating the cartilage segmentation, the main challenges are the thin structure of the cartilage and the low contrast between the cartilage and surrounding soft tissues. The progression of OA is very often slow and it can take many years before the cartilage is reduced from its typical thickness of a few millimeters to possible total loss. It is therefore important to have high accuracy and precision of the cartilage assessment technique in order to detect statistically significant changes [2]. This enables the correlation of the method with the effects of drugs, and the evaluation of their benefit to the joint in reducing the signs of the disease.

MR imaging allows the non-invasive assessment of cartilage tissue, which is required for clinical studies, surgical treatments and drug trials. To obtain statistical significance, this assessment is usually performed on each cartilage separately (or in sub regions) using morphological measures (volume, thickness, surface area or curvature) [4]. These measures require the cartilage to be segmented separately (or in sub regions), a task that can significantly influence the error and reproducibility of the quantitative analysis, and has thus far proved difficult to automate, with current approaches manual or semi-automatic (e.g. region growing, B-spline snakes and live-wires). These approaches take 30 minutes to several hours and require a skilled trained operator to obtain reproducible results. T1 weighted fat suppressed (FS) spoiled gradient recall images (SPGR) are the most commonly used MR sequence for cartilage assessment. They have poor cartilage-meniscus and cartilage-synovial fluid contrast and can exhibit significant imaging artifacts that obscure and artificially create defects [3]. These along with image resolution, magic angle and partial volume effects cause the problems that make accurate cartilage segmentation difficult. A few recently developed sequences reduce many of these problems. In diseased knees, osteophytes, lesions and cracks are commonly observed and make segmentation more difficult. The development of an automatic approach is desirable and has been pursued by several groups. This paper provides a discussion of current segmentation techniques for knee joint from medical imaging such as Computer Tomography (CT) and Magnetic Resonance Imaging (MRI) [1]. Segmentation methods and their applications of knee joint from recent literatures are described and discussed along with the future scope and conclusion.

\section{Motivation}

The knee, one of the most complex and delicate joints in the body, is one of the frequently injured or damaged components of the human motion system. According to the American Academy of Orthopedic Surgeons, the risk of disability from knee problem is as high as that of cardiovascular disease. Osteoarthritis 
(OA) is one of the major health concerns among the elderly today [5]. The main effects of OA is the degradation of the articular cartilage together with remodeling and overgrowth of bone, a process causing loss of mobility of the joints. It typically affects large weight bearing joints as hips and knees. The knee is made up of four major bone segments: the femur, the tibia, the fibula and the patella (knee cap). The knee joint contain soft tissues such as ligaments, cartilages and so on. The ligaments provide stability and strength to the knee joint. Cartilages cover the patella, tibia and femur in the regions where the bones contact one another [6]. Cartilages allow the bones to slide freely on each other.

Currently, the treatment of $\mathrm{OA}$ is restricted to symptom control, because as yet there are no diseasemodifying drugs. Model-based approaches to the interpretation of images of variable objects are now attracting considerable interest. The generation of three-dimensional (3d) knee model from patient-specific medical image data is important for the clinical diagnosis, localization of pathology, treatment planning, and computerintegrated surgery. The reconstructed $3 \mathrm{~d}$ knee model would be used to visualize and analyze the dynamic and kinematic behaviors of the knee joint. Fully automated segmentation of bone and cartilage could be hugely beneficial in clinical trials and in the orthopedic industry [5].

\section{A. Thresholding Approach:}

\section{SEGMENTATION METHODS}

Image segmentation by thresholding is a simple but powerful approach for segmenting images having light objects on dark background. Thresholding technique is based on image space regions i.e. on characteristics of image. Thresholding operation convert a multilevel image into a binary image i.e., it choose a proper threshold $\mathrm{T}$, to divide image pixels into several regions and separate objects from background. Any pixel (x, y) is considered as a part of object if its intensity is greater than or equal to threshold value i.e., $f(x, y) \geq T$, else pixel belong to background [6]. As per the selection of thresholding value, two types of thresholding methods are in existence, Global and Local thresholding. When T is constant, the approach is called Global thresholding otherwise it is called Local thresholding. Global thresholding methods can fail when the background illumination is uneven. In local thresholding, multiple thresholds are used to compensate for uneven illumination. Threshold selection is typically done interactively however, it is possible to derive automatic threshold selection algorithms.

Limitation of thresholding method is that, only two classes are generated, and it cannot be applied to multichannel images. In addition, thresholding does not take into account the spatial characteristics of an image due to this it is sensitive to noise, as both of these artifacts corrupt the histogram of the image, making separation more difficult.

\section{B. Region based Segmentation Methods:}

Compared to edge detection method, segmentation algorithms based on region are relatively simple and more immune to noise. Edge based methods partition an image based on rapid changes in intensity near edges whereas region based methods, partition an image into regions that are similar according to a set of predefined criteria $[7,8,10]$. Segmentation algorithms based on region mainly include following methods:

1. Region Growing: Region growing is a procedure that group's pixels in whole image into sub regions or larger regions based on predefined criterion. Region growing can be processed in four steps:

- Select a group of seed pixels in original image.

- Select a set of similarity criterion such as grey level intensity or color and set up a stopping rule.

- Grow regions by appending to each seed those neighboring pixels that have predefined properties similar to seed pixels.

- Stop region growing when no more pixels met the criterion for inclusion in that region (i.e. Size, likeness between a candidate pixel \& pixel grown so far, shape of the region being grown).

2. Region Splitting and Merging: Rather than choosing seed points, user can divide an image into a set of arbitrary unconnected regions and then merge the regions in an attempt to satisfy the conditions of reasonable image segmentation. Region splitting and merging is usually implemented with theory based on quad tree data. Let $\mathrm{R}$ represent the entire image region and select a predicate $\mathrm{Q}$,

- We start entire image if $\mathrm{Q}(\mathrm{R})=$ FALSE, we divide the image into quadrants, if $\mathrm{Q}$ is false for any quadrant that is, if $\mathrm{Q}(\mathrm{Ri})=$ FALSE, We subdivide the quadrants into sub quadrants and so on till no further splitting is possible.

- If only splitting is used, the final partition may contain adjacent regions with identical properties. This drawback can be remedied by allowing merging as well as splitting i.e. merge any adjacent regions $\mathrm{Rj} \&$ Rk for which, $\mathrm{Q}(\mathrm{Rj} \mathrm{U} \mathrm{Rk})=\mathrm{TRUE}$.

- Stop when no further merging is possible. 


\section{Segmentation based on Artificial Neural Network:}

Neural Network based segmentation is totally different from conventional segmentation algorithms. In this, an image is firstly mapped into a Neural Network. Where every Neuron stands for a pixel, thus image segmentation problem is converted into energy minimization problem. The neural network was trained with training sample set in order to determine the connection and weights between nodes. Then the new images were segmented with trained neural network, for example, we can extract image edges by using dynamic equations which direct the state of every neuron towards minimum energy defined by neural network [8]. Neural network segmentation includes two important steps feature extraction and image segmentation based on neural network.

Feature extraction is very crucial as it determines input data of neural network, firstly some features are extracted from the images, such that they become suitable for segmentation and then they were the input of the neural network. All of the selected features compose of highly non-linear feature space of cluster boundary.

Neural network based segmentation have four basic characteristics:

- Highly parallel ability and fast computing capability, which makes it suitable for real time application.

- Improve the segmentation results when the data deviates from the normal situation.

- Robustness making it insensitive to noise.

- Reduced requirement of expert intervention during the image segmentation process.

However there are some drawbacks of neural networks based segmentation either, such as:

- Some kind of segmentation information should be known beforehand.

- Initialization may influence the result of image segmentation.

- Neural network should be trained using learning process beforehand, the period of training may be very long, and we should avoid overtraining at the same time.

\section{Segmentation based on Clustering:}

Clustering is an unsupervised learning task, where one needs to identify a finite set of categories known as clusters to classify pixels. Clustering use no training stages rather train themselves using available data. Clustering is mainly used when classes are known in advance [11]. A similarity criteria is defined between pixels, and then similar pixels are grouped together to form clusters.

The grouping of pixels into clusters is based on the principle of maximizing the intra class similarity and maximizing the inter class similarity. The quality of a clustering result depends on both the similarity measure used by the method and its implementation. Clustering algorithms are classified as hard clustering, kmean clustering, fuzzy clustering, etc.

\section{E. Deformable Methods:}

Deformable models perhaps are one of the most active and successful segmentation methods. As deformable models are widely used in knee segmentation. A deformable model is a generic description of the object model of interest. The object model deforms iteratively until it matches the information present in the image to be segmented [12]. Deformable models are closed curves or surfaces that deform under the influence of internal and external forces.

The internal forces are defined within the curve or surface to keep the model smooth throughout the deformation. The external forces are designed to drive the model toward an object boundary or desired features within an image. Various deformable models have been proposed based on different deformation mechanisms in the literatures such as snakes, active contours or surfaces, balloons, deformable contours or surfaces, and active shape or appearance models etc. In the snake method, the contour deformation is handled as a mechanical process in which the contour is treated like an elastic band placed in a force field. The snake method requires the initial contour to be close to the target contour and does not allow topological change throughout the contour deformation. Undesired results are produced in handling objects with concave shape or inhomogeneous interior [13]. The level set approach provides a numerical solution to the evolving contour. The method alleviates the limitations of the snake in relaxing initial requirements and being applicability for complex shapes.

Medical experts can provide valuable anatomical knowledge of the organ of interest. Parametric deformable models overcome the deficiencies in those simple segmentation methods through incorporating the a priori knowledge of shape and its variation in the establishment of shapes. Statistical models are used to control the deformation of object model throughout the segmentation. The statistical models are obtained from a number of instance objects that best represent the shape variations. The parametric deformable model methods formulate the boundary searching problem as a maximization of the posteriori probability of a model generated from an input image. Using Bayes rule, the posteriori probability can be transformed into a combination of the model's prior probability and a likelihood function [10]. The likelihood function is formulated by the image features. 
Active shape models (ASM) use the statistical analysis of the shape variation as a priori knowledge to constrain the deformation of $3 \mathrm{~d}$ objects. The prior knowledge of shape is approximated by the Principal Component Analysis (PCA). The obtained vector space represents an alternate shape of the original shape and approximate best the main variations in shapes. The parameters used to estimate the shape is very likely to be Gaussian, thus, a reasonable number of shape and pose parameters would be used to represent the geometry of the constructed object. The ASM method starts with an average model shape, and estimates the new shape iteratively based on the image information. The resulting shape is point distribution model e.g. surface mesh [16]. The computation complexity for ASM method should be done at an acceptable expense while capturing the fine details.

In active appearance models (AAM), a new prior model is constructed using both shape and gray level information. The method is based on the assumption that there exists a linear dependency between the object shape and the intensity pattern in the image around the object vertices. However, when the intensity distribution is influenced by human motion, load deformation etc., the application of AAM segmentation is restricted. AAM is not suitable for the knee joint segmentation due to its basic assumption [15].

Deformable models offer robustness to both image noise and boundary gaps and allow integrating boundary elements into a coherent and consistent mathematical description. With this sense in mind, the deformable model methods, especially the extension methods have potentials in the knee joint segmentation [16].

\section{SEGMENTATION METHODS FOR KNEE JOINTS}

Knee joint image segmentation is a very challenging task because of its complexity. Segmentation methods for knee joint can be classified into three categories based on manual intervention required, namely manual, semiautomatic and fully automatic. The manual segmentation methods are laborious and time consuming [5]. Semiautomatic methods are developed to reduce the manual intervention by automating few steps of processing. Fully automatic methods involve advanced and complex processing steps with certain limitations. Zohara et al. [6] developed a semiautomatic method, initially cartilage is segmented manually by marking the consecutive points along the articular contour curves with a typical spacing of 0.5-1.0 mm. An interpolated cubic B-spline curve is fitted for these points. Cashman et al. [5] developed an algorithm using edge detection and thresholding. Boundary discontinuities are bridged using B-spline interpolation and recursive region growing procedure is used in segmentation of bone. In radial search method developed by Poh et al. [7], a threshold method is used to detect the inner boundaries along the radial lines. The algorithm searches the boundary of cartilage from approximate center of femur bone region. In the method developed by Gamio et al. [8] Bezier splines are used. The control points are placed inside the cartilage following its shape to create a Bezier spline. Rays perpendicular to the spline on the control points are traced to find the bone cartilage interface. The edges are found based on the first derivative of brightness using bicubic interpolation along the line profiles. In the graph cut method developed by Shim et al. [9] seeds are placed manually (curvilinear marks) over specific anatomic regions. The seeds are propagated to neighboring pixels and then segmented. A fully automatic method using voxel classification is developed by Jenny et al. [10]. The algorithm is based on kNN classifier to reduce processing time. In the 2-D active contour algorithm developed by Claude et al. [11], a local coordinate system (LCS) is developed for the femoral and tibial cartilage boundaries for the measurement of thickness and volume. Tang et al. [12] proposed segmentation of articular cartilage surfaces using snakes, and a gradient vector flow (GVF) based external force. GVF snake is made more stable and converge to the correct surfaces, directional gradient is used to produce the gradient vector flow. Segmentation method is developed for multiple interacting surfaces belonging to multiple interacting objects, called LOGISMOS (layered optimal graph image segmentation of multiple objects and surfaces) by Yin et al. [14]. The approach is based on the algorithmic incorporation of multiple spatial inter relationships in a single n-dimensional graph, M S Mallikarjuna Swamy \& Mallikarjun S Holi and followed by graph optimization. Dodin et al. [15] segmented the cartilage by resampling the MRI in the neighborhood of the bone surface. Texture analysis techniques are optimized by filtering and then cartilage is discriminated as a bright and homogeneous tissue. This excludes soft tissues and enables the detection of the external boundary of the cartilage. A Bayesian decision criterion is used for automatic separation of the cartilage and synovial fluid. An unsupervised method is developed for segmentation and quantification of knee features by Tamez Pena et al [16].

Even though there are many algorithms developed for knee joint cartilage segmentation still there is a scope for better segmentation and precise quantification of cartilage because of the complexity involved in segmentation algorithms. 


\section{CONCLUSION AND FUTURE SCOPE}

In this work we have researched the work on knee segmentation from 3D MRI images. We have used active appearance model which has been used in several applications of pattern recognition. Active appearance model was applied to the 2D images of the MRI to segment the knee in each slice of 3D data. However this technique can be easily extended to directly apply active appearance model on 3D MRI data. During search first the coarse scale is used to detect the object which improved efficiency. In this work we described the construction of a volumetric AM to refine the inter-image correspondence provided by surface AAMs. The volumetric AM offers the advantages of not requiring a training set of bone segmentations and enabling regions of high stress, where loss of cartilage is most likely to occur, to be identified using both the shapes of the bones and soft-tissue landmarks visible in the mean model image. The results demonstrate that the volumetric AM approach using 2D surfaces improves the accuracy of the bone segmentations, as measured by deviation from manual segmentations. The volumetric AM also provides robustness and is able to segment smaller structures, such as the patella bone, which was not possible, the results demonstrate that the volumetric AM approach improves the accuracy of the bone segmentations, compared to the surface AAM, as measured by deviation from manual segmentations. The volumetric AM also provides robustness and is able to segment smaller structures, such as the patella bone, which was not possible using the surface based AAM approach. This is due to the volumetric

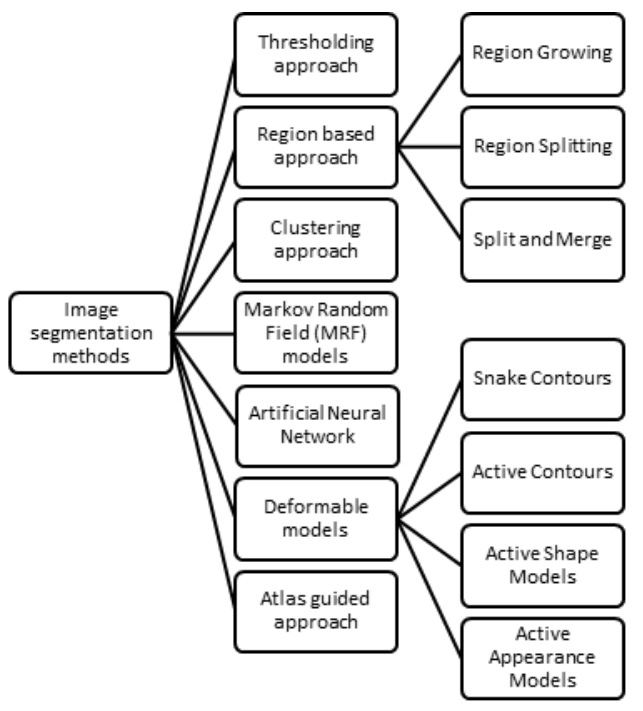

Fig. 1 Various Image segmentation methods

AM's ability to locate the patella in relation to the other structures within the image. Results on reproducibility of regional mean thickness measures indicate that the volumetric AM provides better intra-subject reproducibility across multiple images, indicating that longitudinal measures of change in cartilage thickness are likely to be more sensitive. Further analysis of the study data using the volumetric AM has been reported in concluding that measures of cartilage quality are comparable between vendors' $3 \mathrm{~T}$ scanners, and that findings of studies conducted using Philips or GE scanners are applicable to research on the OAI data, which is acquired on Siemens scanners. Multicenter trails are viable, but the presence of significant bias between scanners suggests that in longitudinal studies, subjects should not be moved between centers.

\section{REFERENCES}

[1] Robert Toth; Anant Madabhushi, Multifeature Landmark-Free Active Appearance Models: Application to Prostate MRI Segmentation, IEEE Transactions on Medical Imaging, vol. 31, no. 8, August 2012.

[2] Martin A. Lindquist; Cun-Hui Zhang; Gary Glover; Lawrence Shepp; Qing X. Yang, A Generalization of the Two-Dimensional Prolate Spheroidal Wave Function Method for Non rectilinear MRI Data Acquisition Methods, IEEE Transactions on Image Processing, vol. 15, no. 9, September 2006.

[3] Wolfgang Wirth; Felix Eckstein, A Technique for Regional Analysis of Femorotibial Cartilage Thickness Based on Quantitative Magnetic Resonance Imaging, IEEE Transactions on Medical Imaging, vol. 27, no. 6, June 2008.

[4] Tomos G. Williams; Automatic Segmentation of Bones and Inter-Image Anatomical Correspondence By Volumetric Statistical Modelling Of Knee MRI, IEEE ISBI 2010

[5] Peter M.M. Cashman., Richard I. Kitney, Munir A.G. and Mary E.C., Automated techniques for visualization and mapping of articular cartilage in MR images of the osteoarthritic knee: a base technique for the assessment of micro damage and sub micro damage, IEEE Trans. Nanobioscience, vol. 1, pp. 42-51, 2002. 
[6] Zohara A. Cohen, Denise M.M., S. Daniel Kwak., Perrine L., Fabian F., Edward J.C., and Gerard A.A., Knee cartilage topography, thickness, and contact areas from MRI: in-vitro calibration and in-vivo measurements, Osteoarthritis and Cartilage, vol.7, pp. 95109,1999

[7] Poh C.L. and Richard I.K., Viewing interfaces for segmentation and measurement results, Proc. of 27th Annual Conf. IEEE Engineering in Medicine and Biology, Shanghai, China, 2005, pp. 5132-5135.

[8] Julio Carballido Gamio, Jan S. Bauer, Keh-Yang Lee, Stefanie Krause and Sharmila Majumdar, Combined image processing techniques for characterization of MRI cartilage of the knee, Proc. 27th Annual Conf. IEEE Engineering in Medicine and Biology, Shanghai, China, 2005, pp.3043-3046.

[9] Hackjoon Shim, Samuel Chang, Cheng Tao, Jin-Hong Wang, C. Kent Kwoh and Kyongtae T. Bae, Knee cartilage: efficient and reproducible segmentation on high spatial-resolution MR images with the semi-automated graph-cut algorithm method, Radiology, vol. 251 , pp. 548-556, 2009.

[10] Jenny F., Erik B.D., Ole F.O., Paola C.P. and Claus C., Segmenting articular cartilage automatically using a voxel classification approach, IEEE Trans. Medical Imaging, vol. 26, pp.106-115, 2007.

[11] Claude K., Pierre G., Benoît G., Alain G., Gilles B., Jean P.R., Johanne M.P., Jean Pierre Raynauld, Johanne M.P., Jean Pierre P. and Jacques A. de G., Computer aided method for quantification of cartilage thickness and volume changes using MRI: validation study using a synthetic model, IEEE Trans. Biomedical Engineering, vol. 50, pp. 978-988, 2003.

[12] Jinshan Tang, Steven Millington, Scott T. Acton, Jeff Crandall, and Shepard Hurwitz, Surface extraction and thickness measurement of the articular cartilage from MR images using directional gradient vector flow snakes, IEEE Trans. Biomedical Engineering, vol. 53, pp.896-907, 2006.

[13] Yin Yin, Xiangmin Zhang, Rachel Williams, Xiaodong Wu, Donald D. Anderson and Milan Sonka, LOGISMOS-Layered Optimal Graph Image Segmentation of Multiple Objects and Surfaces: cartilage segmentation in the Knee Joint, IEEE Trans. Medical Imaging, vol. 29, pp. 2023-2037, 2010.

[14] Pierre Dodin, Jean Pierre Pelletier, Johanne Martel Pelletier and François Abram, Automatic human knee cartilage segmentation from 3D magnetic resonance images, IEEE Trans. Biomedical Engineering, vol. 57, pp. 2699-2711, 2010.

[15] Meenaz H. Shaikh, Anuradha Joshi, Sharddha Panbude, Inter-image Anatomical Correspondence and Automatic Segmentation of Bones by Volumetric Statistical Modelling of Knee MRI, International Journal of Computational Engineering Research, vol. 04, pp. 52-53, 2014.

[16] Jose G. Tamez Pena, Joshua Farber, Patricia C. Gonzalez, Edward Schreyer, Erika Schneider, and Saara Totterman, Unsupervised segmentation and quantification of anatomical knee features: Data from the Osteoarthritis Initiative, IEEE Trans. Biomedical Engineering, vol. 59, pp.1177-1186, 2012. 